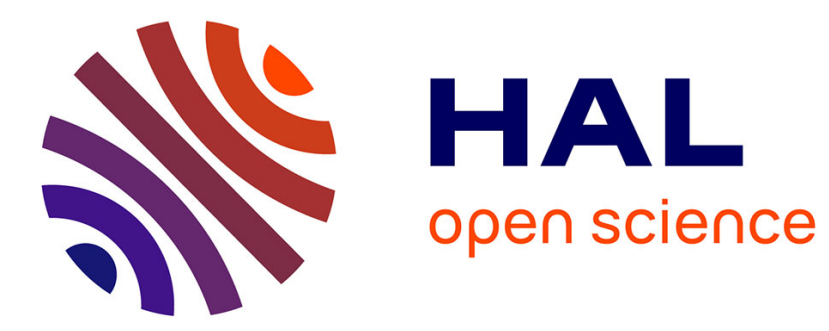

\title{
La gestion des émotions au travail : le cas des policiers d'élite
}

\author{
Hélène Monier
}

\section{To cite this version:}

Hélène Monier. La gestion des émotions au travail: le cas des policiers d'élite. RIMHE: Revue Interdisciplinaire Management, Homme(s) \& Entreprise, 2014, 13, pp.105 à 121. hal-01066074

\section{HAL Id: hal-01066074 https://univ-lyon3.hal.science/hal-01066074}

Submitted on 19 Sep 2014

HAL is a multi-disciplinary open access archive for the deposit and dissemination of scientific research documents, whether they are published or not. The documents may come from teaching and research institutions in France or abroad, or from public or private research centers.
L'archive ouverte pluridisciplinaire HAL, est destinée au dépôt et à la diffusion de documents scientifiques de niveau recherche, publiés ou non, émanant des établissements d'enseignement et de recherche français ou étrangers, des laboratoires publics ou privés. 


\title{
LA GESTION DES ÉMOTIONS AU TRAVAIL : LE CAS DES POLICIERS D'ÉLITE
}

\author{
Hélène Monier
}

ARIMHE | RIMHE : Revue Interdisciplinaire Management, Homme(s) \& Entreprise

2014/4 - $\mathrm{n}^{\circ} 13$

pages 105 à 121

ISSN 2259-2490

Article disponible en ligne à l'adresse:

http://www.cairn.info/revue-rimhe-2014-4-page-105.htm

Pour citer cet article :

Monier Hélène, «La gestion des émotions au travail : le cas des policiers d'élite »,

RIMHE : Revue Interdisciplinaire Management, Homme(s) \& Entreprise, 2014/4 n 13, p. 105-121.

Distribution électronique Cairn.info pour ARIMHE.

(c) ARIMHE. Tous droits réservés pour tous pays.

La reproduction ou représentation de cet article, notamment par photocopie, n'est autorisée que dans les limites des conditions générales d'utilisation du site ou, le cas échéant, des conditions générales de la licence souscrite par votre établissement. Toute autre reproduction ou représentation, en tout ou partie, sous quelque forme et de quelque manière que ce soit, est interdite sauf accord préalable et écrit de l'éditeur, en dehors des cas prévus par la législation en vigueur en France. II est précisé que son stockage dans une base de données est également interdit. 


\title{
La gestion des émotions au travail: le cas des policiers d'élite
}

\section{Hélène MONIER ${ }^{1}$}

\begin{abstract}
Résumé
Les métiers de la police impliquent un travail émotionnel important (Hochschild, 2003). Pour les policiers d'élite, ce dernier est nécessaire afin de mener à bien la mission, mettant en danger leur intégrité physique et/ou mentale. Leurs compétences émotionnelles leur permettent d'effectuer ce travail émotionnel, et de réguler leurs émotions en intervention. Cet article met en relation travail émotionnel, compétences émotionnelles, régulation émotionnelle et soutien social, concernant la prise en compte du facteur émotionnel au travail dans les unités d'élite de la police. Cette étude exploratoire comprend trois niveaux d'analyse: le niveau individuel, le niveau collectif et managérial, et le niveau des pratiques instituées de Gestion des Ressources Humaines (GRH). Comment les pratiques de Gestion des Ressources Humaines des métiers de la police d'élite prennent-elles en compte les compétences émotionnelles (Salovey et Mayer, 1990) qui permettent le travail émotionnel des policiers? La santé du policier et la qualité des interventions sont en jeu. Des policiers d'unités d'élite, la plupart très reconnus et retirés des services, ont été interrogés. Tous les policiers consultés rationnalisent le danger consciemment, pour maîtriser leurs émotions, et se conditionner au calme. Cette réévaluation cognitive (Gross, 1998) leur permet de faire concorder au maximum l'émotion affichée avec l'émotion ressentie: le travail émotionnel semble s'effectuer en profondeur. Au niveau collectif, les policiers insistent sur le rôle bénéfique de plusieurs formes de soutien social. Enfin, concernant les pratiques instituées de GRH, les émotions sont prises en compte surtout dans le recrutement. La gestion des émotions se développe davantage au cours des entraînements quotidiens, en conditions proches du réel que dans le cadre de sessions de formation explicitement dédiées à la gestion des émotions. Des préconisations managériales se profilent, au-delà de ces deux pratiques de GRH que sont le recrutement et la formation : prendre en compte les émotions au travail dans la gestion des carrières et des retraits (absentéisme, réaffectations de poste, rotations des postes, autres retraits) et instaurer des conditions favorables permettant le soutien social. Notons quelques étonnements et pistes nouvelles ayant émergé de cette étude: au-delà des compétences émotionnelles, la "foi », l'entraînement, et le rôle de l'humour, permettraient aux professionnels de réguler leurs émotions; et une addiction à l'adrénaline pourrait influencer la gestion des carrières.
\end{abstract}

Mots-clés: émotions, compétences émotionnelles, travail émotionnel, régulation émotionnelle, soutien social, pratiques de gestion des ressources humaines.

1 - Doctorante, Sciences de Gestion, Université Jean Moulin Lyon III, Centre de Recherche Magellan (EA 3713) - helene.monier@gmail.com 


\begin{abstract}
Police elite units involve significant emotional labor (Hochschild, 2003). For elite police officers, this emotional labor is necessary to carry out the mission, endangering their physical and/or mental health. Their emotional competencies allow them to do this emotional labor, and regulate their emotions during an intervention. This article put in relation emotional labor, emotional competencies, emotional regulation and social support, taking into account the emotional factor at work in the elite police units. This exploratory study includes three levels of analysis: the individual level, the collective and managerial level, and the level of institutional practices of Human Resources Management (HRM). How do the HRM practices of elite police units take into account the emotional competencies (Salovey and Mayer, 1990) allowing the hard emotional labor of the police officers? Both the professionals' health and the intervention's quality depend on it. Some police officers in these units, most of them highly recognized and removed from service, were interviewed. All of them streamline the danger, consciously, to control their emotions and to condition themselves to be calm. This cognitive reappraisal (Gross, 1998) permits them to match up the displayed emotion with the really felt emotion: emotional labor appears to be an in-depth action. At the collective level, police officers insist on the beneficial role of various forms of social support. Finally, concerning the institutional practices of HRM, emotions are taken into account especially in recruitment. Managing emotions is developed further during daily training, in conditions close to the reality, then as part of training sessions specifically dedicated to the management of emotions. Managerial recommendations emerge beyond these two HRM practices: taking into account emotions at work in the careers and withdrawals management (absenteeism, job reassignments, job rotations and other withdrawals) and creating favorable conditions for social support. We note a few surprises and new tracks having sprung from this study: beyond the emotional competencies, "faith", trainings, and the role of humor, will allow the professionals to regulate their emotions; and an addiction to adrenaline could influence the career management.
\end{abstract}

Key-words : emotions, emotional competencies, emotional labor, emotion regulation, social support, practices of human resources management. 


\section{Introduction}

Générée par un objet ou un événement (Lazarus, 1991), l'émotion comporte cinq éléments: la cognition, la physiologie, l'expression motrice, les tendances à l'action et le sentiment subjectif (Scherer, 2000). L'organisme répond à l'évaluation d'un stimulus interne ou externe (Scherer, 2000, p.139). Le travail émotionnel se définit comme un acte qui vise à changer le degré ou la qualité d'une émotion, afin de s'ajuster à une situation (Hochschild, 2003). Pour certains métiers, il fait partie intégrante du travail attendu (Hochschild, 2003): c'est le cas des métiers de la police (Loriol, 2003). Afin d'effectuer ce travail émotionnel, les policiers et leurs managers développent un certain nombre de compétences émotionnelles (Salovey et Mayer, 1990). Ces dernières peuvent être évaluées et prises en compte dans la Gestion des Ressources Humaines (GRH) au niveau du recrutement, de la formation, des formes de coopération et de management de proximité au sein des groupes restreints de travail, ainsi qu'au niveau de leurs répercussions éventuelles sur la santé des professionnels. Les unités d'élite étudiées sont les suivantes: le RAID (Recherche - Assistance - Intervention Dissuasion), le GIPN (Groupe d'Intervention de la Police Nationale), la BRI (Brigade de Recherche et d'Intervention), et d'autres services qui exigent de ne pas être cités. Comment les pratiques de GRH de ces unités d'élite prennentelles en compte les compétences émotionnelles des professionnels, permettant l'important travail émotionnel? Sur le plan théorique, cette recherche vise deux objectifs majeurs. Dans un premier temps, il s'agit d'articuler le travail émotionnel (Hochschild, 2003) et la régulation émotionnelle (Gross, 1998). En effet, les deux types de travail émotionnel proposés par Hochschild (2003), travail en surface et travail en profondeur, s'associent, en observation de situation de travail, à deux composantes de la régulation émotionnelle (Gross, 1998): suppression expressive et réévaluation cognitive. Dans un second temps, travail émotionnel (Hochschild, 2003) et compétences émotionnelles (Salovey et Mayer, 1990, 1997) s'avèrent pertinents à mettre en relation du point de vue de la GRH: en contexte de travail, ce sont les compétences émotionnelles du professionnel qui lui permettent d'effectuer le travail émotionnel requis par l'organisation. En situation de travail, l'un ne va pas sans les autres. Au niveau managérial, la prise en compte de la gestion émotionnelle en intervention dans trois niveaux d'analyse, GRH, management de proximité et professionnels sur le terrain, a une incidence sur deux aspects organisationnels : la santé du professionnel d'une part, et la qualité des interventions de l'autre. L'absentéisme, les départs, la mauvaise ambiance, le délitement social, le turn-over ont un coût. La qualité de l'intervention se trouve affectée par une baisse de la concentration ou d'attention du professionnel, causée par l'insuffisante qualité du travail émotionnel fourni (Van Hoorebeke, 2004). Les conséquences d'une non-prise en compte des aspects émotionnels dans ces métiers (travail émotionnel fourni et compétences émotionnelles requises) peuvent s'avérer dramatiques, à la fois au niveau humain et individuel, et au niveau de l'organisation et de sa performance collective (Van Hoorebeke, 2004). Dans la première partie de l'étude, la revue de littérature met en relation travail émotionnel et régulation émotionnelle. Les stratégies de mise en place et de facilitation du travail émotionnel ne s'appliquent que si la personne dispose de compétences émotionnelles, gérées par l'individu, par le management, 
et par la GRH. Ensuite, cette revue de littérature traitera des types de soutien social nécessaires dans ces métiers. Concernant l'axe méthodologique, il s'agit d'une étude exploratoire avec analyse de données primaires et secondaires. Des entretiens semi-directifs (Blanchet et al, 1992) dans plusieurs unités d'élite constituent les données primaires principales (Hamel, 1993; Hamel, 1997; Rispal, 2002; Yin, 2002). La troisième partie présente et discute les résultats de cette recherche, et permet de repérer les pratiques de gestion émotionnelle dans les unités. La discussion des résultats aboutit à la découverte de nouveaux aspects de gestion émotionnelle, non pris en compte dans la littérature: il s'agit du rôle de la « foi » en intervention, de l'entraînement ou " mithridatisation », et de l'addiction aux situations extrêmes. Enfin, de nouvelles pistes de recherche sont envisagées.

\section{De l'individu au collectif : les émotions au travail articulent quatre concepts}

Nous considérons que les émotions au travail articulent quatre concepts interdépendants, examinés l'un après l'autre: le travail émotionnel, les compétences émotionnelles, la régulation émotionnelle. Ces trois premiers concepts concernent l'individu pris isolément dans sa pratique professionnelle. La question du soutien social participe également de la gestion des émotions au travail. Elle met davantage en jeu les interactions qui se nouent au sein des équipes d'intervention.

\subsection{Le travail émotionnel}

Rappelons que le travail émotionnel est un acte qui vise à changer le degré ou la qualité d'une émotion, afin de s'ajuster à une situation (Hochschild, 2003). Dans certains métiers, l'émotion n'est pas spontanée, mais interprétée, contrôlée, réprimée ou suscitée. Par exemple le policier d'élite, va devoir dégager une attitude de calme, de maîtrise de la situation, voire de neutralité, pendant l'intervention. S'il n'effectue pas ce travail émotionnel, les conséquences pourront être dramatiques: échec de l'intervention, décès du professionnel, d'un collègue, ou du forcené, délitement du groupe d'intervention, etc. Il existe deux formes de travail émotionnel: un travail émotionnel en surface, appelé jeu superficiel, et un travail émotionnel en profondeur, appelé jeu en profondeur (Hochschild, 2003). Tout d'abord, le jeu superficiel, ou surface acting, consiste à feindre des émotions non réellement ressenties. Selon Adelmann (1995), ce jeu est le plus stressant et représente un risque psychosocial, car il y a dissonance émotionnelle (Van Hoorebeke, 2003). En revanche, le jeu en profondeur, ou deep acting, nécessite de chercher à ressentir vraiment l'émotion exprimée. Le professionnel va faire correspondre au maximum l'émotion affichée avec l'émotion ressentie. Le travail émotionnel implique mentalement l'individu: il nécessite donc des retraits. Par exemple, après une intervention à forte charge émotionnelle, le professionnel va devoir rester au calme, effectuer d'autres tâches moins risquées : certains policiers doivent rendre un rapport écrit post-intervention, prendre des jours de repos (rotations de postes), le temps que la charge émotionnelle et ses conséquences disparaissent ou s'atténuent. Les professionnels se protègent individuellement grâce à des stratégies de retrait, d'évitement, de distanciation, en cas d'émotion trop forte ou de travail émotionnel trop important. Les formes de travail 
émotionnel permettent d'explorer les moyens d'adaptation des professionnels en contexte de travail, lors de fortes charges émotionnelles. En réponse, et pour s'y adapter, le professionnel élabore des stratégies de régulation émotionnelle afin de se protéger.

\subsection{La régulation émotionnelle}

Selon Gross (1998), il y aurait cinq séquences de régulation des émotions: la sélection de la situation, la modification de la situation, le déploiement attentionnel, la réévaluation cognitive, et la suppression expressive. Nous analyserons plus particulièrement les deux dernières séquences de la régulation émotionnelle: la réévaluation cognitive et la suppression expressive, ces séquences ayant la particularité de pouvoir être mises en relation avec les deux formes de travail émotionnel. D'une part, la réévaluation cognitive consiste, pour le professionnel, à réévaluer la situation et les émotions qui lui sont liées. Il reconsidère la signification émotionnelle d'un événement (Gross, 2001; Gross, 2002). Par exemple, un policier d'élite va se focaliser sur des solutions et non pas sur le péril, ce qui fera baisser l'intensité de sa peur. D'autre part, lors de la suppression expressive, l'individu modifie au moins une des composantes de la réponse émotionnelle de deux façons possibles: soit il supprime l'expression de son émotion ressentie réellement - par exemple montrer de la neutralité alors qu'on ressent de la colère -, soit il affiche une autre émotion que celle réellement ressentie - par exemple montrer de la joie par un sourire, alors que l'émotion ressentie réellement est de la colère. La régulation émotionnelle peut être consciente ou non. Néanmoins, lors d'un travail émotionnel, elle sera la plupart du temps consciente (Gross et al, 2006). La différence principale entre ces deux composantes de la régulation émotionnelle, réside en ce que pour la première, l'individu agit mentalement et physiquement sur sa conscience de la situation et des émotions qui sont en jeu, afin de ressentir réellement l'émotion adaptée et attendue de lui; alors que pour la seconde, l'individu va uniquement agir sur son expression faciale et corporelle pour feindre une émotion, ou de la neutralité. La réévaluation cognitive peut s'apprendre, s'acquérir. Le préjudice sur la santé sera moins important que dans le cas de la suppression expressive (Gross et John, 2003). Afin de réguler leurs émotions au travail, les professionnels utilisent leurs compétences émotionnelles. Ces dernières se divisent en quatre sous-compétences théoriquement mesurables.

\subsection{Les compétences émotionnelles}

Nous analyserons ici les compétences émotionnelles des professionnels suivant le modèle des compétences émotionnelles théorisé par Salovey et Mayer (1990, 1997, 1999, 2004). Ce modèle prend en compte les compétences émotionnelles en tant qu'habiletés mentales, démontrables et mesurables (Salovey et Mayer, 1997; Salovey et al., 2004; Kotsou, 2008). Ce modèle comprend quatre aptitudes, développées dans le tableau 1. Pour ces quatre dimensions, on différencie les compétences émotionnelles intra-personnelles (la gestion émotionnelle par rapport à soi), des compétences émotionnelles interpersonnelles (la gestion émotionnelle par rapport aux autres). Salovey et Mayer (1990) estiment que ces compétences peuvent être mesurées par des tests de performance, comme 
c'est déjà le cas du QI (Quotient Intellectuel). Selon eux, les personnes à fort QE (Quotient Emotionnel) se montrent plus efficaces socialement et au travail, en fonction des missions de l'individu: en particulier concernant le leadership, et aussi pour tout contact avec un public. Salovey et Mayer (1990, p.189) ont défini le QE comme « la mesure d'une forme d'intelligence qui suppose la capacité à contrôler ses sentiments et émotions et ceux des autres, et utiliser cette information pour orienter ses pensées et ses gestes ». Ainsi, il semble que les informations émotionnelles puissent être détectées et traitées de manière consciente et réfléchie. D'après Ekman (2010), former les policiers à la reconnaissance du langage nonverbal, est non seulement possible, mais nécessaire, pour une meilleure qualité des interventions, par une meilleure gestion du travail émotionnel, grâce à un développement des compétences émotionnelles. Ces compétences agissent sur les plans interpersonnel et intra-personnel. Il s'agit de différencier deux compétences: la détection et l'interprétation de la communication non-verbale d'autrui (le forcené par exemple), et le contrôle de sa propre communication non-verbale (contrôle des expressions faciales, ne pas montrer de signes de peur, d'anxiété, etc), qui n'est rien de moins que du travail émotionnel. Les formes de travail émotionnel, les composantes de la régulation émotionnelle et les compétences émotionnelles représentent des stratégies de gestion émotionnelle individuelle. Le soutien social apparaît comme une stratégie de gestion émotionnelle collective.

Tableau 1: Les compétences émotionnelles

suivant le modèle de Salovey et Mayer (1997, p.11)

\begin{tabular}{|l|l|}
\hline $\begin{array}{l}\text { Compétences } \\
\text { émotionnelles } \\
\text { intra- personnelles }\end{array}$ & $\begin{array}{l}\text { - la perception émotionnelle, ou habileté à percevoir et à exprimer les } \\
\text { émotions (perception, appraisal and expression of emotion); } \\
\text { - l'assimilation émotionnelle, comme facilitation émotionnelle dans la pensée } \\
\text { (emotional facilitation of thinking); } \\
\text { - la compréhension émotionnelle, ou habileté à comprendre et raisonner au } \\
\text { sujet d'émotions complexes (understanding and analysing emotions) }\end{array}$ \\
\hline $\begin{array}{l}\text { Compétences } \\
\text { émotionnelles } \\
\text { interpersonnelles }\end{array}$ & $\begin{array}{l}\text { - la gestion des émotions comme habileté à gérer ses émotions et celles } \\
\text { d'autrui (reflective regulation of emotions, managing emotions) }\end{array}$ \\
\hline
\end{tabular}

\subsection{Le soutien social}

Dans les métiers de la police, une juste distance doit s'établir entre le professionnel et les personnes avec lesquelles il est en contact (le forcené pour le négociateur par exemple). Il s'agit de se préserver (Fernandez et al, 2008). Molinier (1998) précise à ce sujet que plaisanter est le meilleur moyen de prendre du recul face au travail émotionnel intense. Le soutien social intervient comme facilitateur de la régulation émotionnelle et du travail émotionnel. Il revêt deux aspects: le soutien social professionnel, qui représente le soutien social de référence, et le soutien social hors travail, ou socio-émotionnel. Ce soutien socio-émotionnel existe la plupart du temps grâce aux amis, à la famille, et aux groupes sociaux. Le soutien social professionnel peut provenir, certes, des collègues, mais surtout de la hiérarchie (Cintas et Sprimont, 2011), sinon, l'auto-régulation, ou maîtrise des émotions du groupe, peine à se mettre en place (Ruiller, 2010). Face à des situations de violence, le soutien social professionnel est un moyen de gérer le stress (La Rocco et Jones, 1978). Etre capable d'échanges sociaux 
appropriés représente une compétence émotionnelle précieuse dans les métiers à risques. Il convient de préciser que le soutien social reste un enjeu très fort de gestion émotionnelle dans la mesure où il s'ancre dans une véritable culture professionnelle, comme chez les policiers (Fernandez et al., 2008). La plupart du temps, chez ces derniers, il existe des groupes de parole informels. Cette revue de littérature présente les formes de travail émotionnel, certaines composantes de la régulation émotionnelle, les compétences émotionnelles principales des professionnels, et le rôle du soutien social dans la gestion des émotions au travail. Nous pouvons alors nous poser la question du lien entre ces quatre concepts. La régulation émotionnelle s'inscrit dans le travail émotionnel. Les compétences émotionnelles servent le travail émotionnel. Le soutien social, quant à lui, facilite la gestion émotionnelle en général. L'approche gestionnaire de cette étude consiste à considérer ces quatre concepts, dans l'objectif de favoriser la qualité des interventions, tout en préservant la santé du professionnel.

\section{Etude empirique: le cas des policiers d'élite}

Afin de détecter la prise en compte des compétences émotionnelles et du travail émotionnel des professionnels dans les unités étudiées, des données d'étude exploratoire, relevant de la GRH, du management de proximité et du terrain, ont été collectées. Les unités d'élite représentant des " cas d'école » de situations à fortes charges émotionnelles, nous avons pu observer les différents niveaux de prise en compte des émotions: au niveau individuel, collectif, et de GRH. Sept entretiens semi-directifs ont été menés en face à face, de deux à quatre heures chacun.

Tableau 2: Principales caractéristiques des professionnels interrogés

\begin{tabular}{|c|c|c|c|}
\hline Unité étudiée & \multicolumn{2}{|c|}{ Caractéristiques clés } & Rôle dans l'étude de terrain \\
\hline $\begin{array}{c}\text { Experts des } \\
\text { compétences } \\
\text { émotionnelles }\end{array}$ & $\begin{array}{c}\text { Docteur en } \\
\text { Psychologie du } \\
\text { travail }\end{array}$ & $\begin{array}{c}\text { Docteur en } \\
\text { Comportements } \\
\text { Organisationnels }\end{array}$ & Elaboration des guides d'entretien \\
\hline RAID & $\begin{array}{c}\text { Ancien } \\
\text { négociateur, } \\
\text { formateur et } \\
\text { recruteur au } \\
\text { RAID } \\
\text { négociateur en } \\
\text { chef, formateur } \\
\text { et recruteur au } \\
\text { RAID } \\
\text { Manager } \\
\text { d'opérationnels }\end{array}$ & $\begin{array}{c}\text { Manager de } \\
\text { négociateurs }\end{array}$ & $\begin{array}{c}\text { Données secondaires: vidéos d'interventions } \\
\text { confidentielles, documentations issues des } \\
\text { formations, archives professionnelles et } \\
\text { personnelles, analyse d'ouvrages, observation } \\
\text { de formation au combat et à la gestion du } \\
\text { stress lors d'attaques avec arme }\end{array}$ \\
\hline BRI & $\begin{array}{c}\text { Chef du service opérationnel } \\
\text { Opérationnel de terrain et fonctions } \\
\text { de management }\end{array}$ & $\begin{array}{c}\text { Données primaires d'entretien } \\
\text { Données secondaires : analyse d'ouvrage de } \\
\text { professionnel }\end{array}$ \\
\hline GIPN & $\begin{array}{c}\text { Retiré des services. Ancien } \\
\text { négociateur et opérationnel de } \\
\text { terrain }\end{array}$ & $\begin{array}{c}\text { Données primaires d'entretien } \\
\text { Données secondaires: reportages vidéo }\end{array}$ \\
\hline Autres & $\begin{array}{c}\text { Retiré des services. Professionnel } \\
\text { Services terrain spécialiste des zones de } \\
\text { guerre }\end{array}$ & $\begin{array}{c}\text { Données primaires d'entretien } \\
\text { Données secondaires: documents } \\
\text { professionnels et personnels, présence aux } \\
\text { formations en auditeur libre }\end{array}$ \\
\hline
\end{tabular}


Les retranscriptions intégrales des entretiens ont été validées par les participants. Suite à une démarche itérative, les données de terrain ont été réduites et compressées (Allard-Poesi, 2003) afin de créer du sens et de procéder à la théorisation (Giroux, 2003). L'analyse des résultats s'est faite par codage manuel. Les informations ont été classées en tableaux, simplifiées, comparées, afin de générer des conclusions. Les données pertinentes sont sélectionnées, mises en relation, puis synthétisées. Les tableaux des résultats en annexes présentent les verbatims extraits des entretiens retranscrits, afin d'illustrer les différents niveaux d'appréhension des émotions. Nous discuterons ensuite des formes de gestion émotionnelle non traitées dans la revue de littérature mentionnées par les policiers interrogés.

\subsection{Analyse des résultats et interrelations de concepts}

Les résultats de l'étude sont discutés à la lumière des trois niveaux d'analyse: le niveau individuel, le niveau collectif et celui de la fonction RH. Ces résultats établissent des liens entre travail émotionnel et régulation émotionnelle, et entre travail émotionnel et compétences émotionnelles. Au niveau individuel, les professionnels sous-entendent que le travail émotionnel s'exerce la plupart du temps en profondeur, et non en surface, c'est-à-dire qu'ils ressentent le plus possible l'émotion qu'ils montrent. Par exemple, le négociateur du RAID se conditionne au calme, se persuade que la situation n'est pas extraordinaire, afin de pouvoir dégager ce calme avec authenticité: "Je me conditionne au calme» (RAID). Pour effectuer ce travail en profondeur, et gérer les émotions en intervention, les policiers réévaluent cognitivement la situation, la plupart du temps de manière consciente (Gross et al., 2006), afin de maîtriser leur peur en intervention, et dégager une attitude qui corresponde avec ce qui est attendu d'eux de la part du public rencontré, de l'équipe, et de la hiérarchie: "Quand il y a des coups de feu... je dois renvoyer une image de calme et de maîtrise. Du coup, je suis convaincu intérieurement que je suis dans cette condition de calme. Si c'eût été un jeu en surface, c'eût été décelable » (RAID). Le témoignage des professionnels confirme l'imbrication entre travail émotionnel en profondeur et réévaluation cognitive. Le professionnel aura tendance à faire coïncider au maximum les émotions: affichée et ressentie. Dans ce cas, il n'y a pas de dissonance émotionnelle, et les impacts sur la santé s'en trouvent moindres que lors d'un jeu en surface (Adelmann, 1995). Cette réévaluation est donc fondamentale pour les professionnels et va permettre d'effectuer le jeu en profondeur, garantir un maximum d'authenticité de l'émotion, et donc une meilleure qualité de l'intervention, car le forcené va détecter la sincérité de l'émotion affichée (Ekman, 2010). Face à des différences de degré dans le travail émotionnel, quel lien peut-on établir avec la santé du professionnel? Ce lien n'est pas toujours évoqué par les professionnels. Pour certains, ce travail émotionnel leur «coûte », pour d'autres, non. Or, la revue de littérature faisait état d'une relation certaine entre travail émotionnel en profondeur et préservation de la santé du professionnel (Adelmann, 1995) par l'atténuation des dissonances émotionnelles (Van Hoorebeke, 2003). Ayant mis en relief l'importance du lien entre la réévaluation cognitive et le travail émotionnel en profondeur, nous analysons comment ce dernier s'articule avec le concept de compétences émotionnelles.

La revue de littérature souligne l'intérêt de former les agents de sécurité à la 
reconnaissance du langage non-verbal (Ekman, 2010). L'observation confirme ce point: les compétences émotionnelles contribuent en tant que moyen au travail émotionnel en profondeur. Pour adapter leur comportement à ce qui est attendu d'eux, les policiers d'élite travaillent sur la communication non-verbale, moyen de gestion émotionnelle et de détection émotionnelle: "Il va falloir que vous sortiez de votre personne tout en restant dedans » (BRI). Les expressions faciales étant universelles, il est possible de les lire, et d'apprendre à contrôler la communication non-verbale: reconnaître les émotions du forcené, et gérer sa communication nonverbale face à un public particulier. Il s'agit ici de compétences à la fois intrapersonnelles et interpersonnelles: "Immanquablement, nous finissions par voir passer une, deux ou trois personnes, dont l'attitude, le comportement général, nous faisaient nous retourner l'un vers l'autre et hocher de la tête: nous avions découvert nos cibles » (GIPN). Il semblerait qu'un processus collectif intervienne également dans la gestion des émotions, le soutien social. Le soutien social présenté dans la revue de littérature revêt deux formes principales: le soutien social de la hiérarchie (Cintas et Sprimont, 2011) et celui des collègues. Les témoignages des policiers affirment que le soutien social principal provient des collègues. Ce soutien participe de l'esprit d'équipe et de débriefes quasiment automatiques post-intervention, plus ou moins formels (Fernandez et al., 2008): «Le sas de décompression et de cohésion se fait généralement au restaurant après une opération. Ce sas est quelque chose qui ne peut être évité » (autres services de sécurité de l'Etat). Ce soutien social permet aux policiers de réguler leurs émotions et de gérer leur stress avant, pendant et après l'intervention (La Rocco et Jones, 1978): "Je suis bien parce que le fait que tout le monde me regarde et craigne pour moi fait que je me sentais bien » (RAID, durant une mise en joue par le forcené). Par contraste, les policiers déplorent un manque de soutien social de la part de la haute hiérarchie: "Parfois, de la part de notre Direction, on a une volonté de ne pas venir nous féliciter...»(BRI). Tous constatent l'action bénéfique du soutien social sur le travail émotionnel et la régulation émotionnelle. Le soutien social agit comme collectif fondamental pour aider les professionnels à accomplir leur travail émotionnel, et supporter les charges émotionnelles dans le cadre de leurs missions. D'autres formes de soutien social émergent: l'humour et la reconnaissance sociale. L'humour, peu présent dans la revue de littérature (Molinier, 1998), constitue cependant une forme de soutien social: " C'est une manière de décompresser » (BRI). Il autorise la distance et le recul face à des événements chargés émotionnellement. L'importance de bien cloisonner vie professionnelle et vie personnelle apparait lors des témoignages des policiers: "On cloisonne. C'est le maître mot 》 (autres services de sécurité de l'Etat). La vie privée apparaît alors comme donnant un cadre de stabilité, qui permet au professionnel de reconstituer ses forces émotionnelles, de se « reposer » émotionnellement. Ce clivage essentiel ressort peu dans la revue de littérature. Celle-ci n'insiste pas non plus sur la reconnaissance sociale. Cette dernière représente pourtant un soutien social important, dans le sens où ces métiers d'élite sont reconnus et valorisés par la société: "Ils me disent que ce que je fais est le métier dont ils rêvaient »(BRI).

Au niveau de la GRH, certains outils, pratiques, ou conditions de travail particulières peuvent encourager les formes de gestion émotionnelle individuelle et collective. Le recrutement et le développement des compétences émotionnelles constituent les deux principales pratiques de GRH repérées. Les professionnels de la fonction $\mathrm{RH}$ 
détectent les compétences émotionnelles des policiers lors des recrutements. Ces compétences se développent ensuite lors des formations et des entraînements. Ces métiers nécessitent un état physique hors pair, lié au niveau de contrôle émotionnel. En effet, pour ces professionnels, le physique et l'émotionnel s'imbriquent étroitement: "Toutes les phases de préparations, d'exercices et d'entrainements sont poussées » (autres services de sécurité de l'Etat). Il est ainsi possible de voir, par des mises en situation très physiques, le niveau de contrôle émotionnel et de gestion du stress du candidat. De cette manière, et par différentes formes de tests (psychologiques, physiques, de groupe, de management), les recruteurs peuvent identifier, avec l'aide d'un psychologue, les compétences émotionnelles des candidats. Nous retrouvons, lors des tests de recrutement, de manière non formalisée, les quatre habiletés du modèle de Salovey et Mayer (1990): percevoir et exprimer les émotions (tests d'agressivité, sang-froid, simulations), la facilitation émotionnelle dans la pensée (prises de décisions, initiatives), la compréhension et l'analyse des émotions (adaptation, discernement, mises en situation), et la gestion des émotions (maîtrise de soi, compétences interpersonnelles, empathie) (Salovey et Mayer, 1990). Ces quatre habiletés regroupent des compétences émotionnelles interpersonnelles et intra-personnelles. Concernant les formations, les négociateurs d'élite bénéficient de formations très poussées, avec entre autres des aspects relevant de la gestion émotionnelle en intervention. En revanche, les policiers opérationnels de terrain déplorent un manque de formation développant leurs compétences émotionnelles: "Je n’ai jamais assisté à un cours de gestion du stress au RAID! » (RAID).

\subsection{L'évocation de la "foi», de la "mithridatisation» et de l'addiction aux situations extrêmes}

Des stratégies de régulation émotionnelle et de travail émotionnel ont émergé lors des entretiens. Les policiers interrogés ont amené l'idée que la «foi » leur permettait de passer à l'action, d'effectuer le travail émotionnel nécessaire, et de réguler les émotions fortes.

Tableau 3: La « foi », comme moyen de gestion émotionnelle des policiers

\begin{tabular}{|l|l|}
\hline BRI & $\begin{array}{l}\text { "On croit dans ce qu'on fait. Vous ne pouvez pas y aller, monter sur des terroristes si vous } \\
\text { n'avez pas une notion de foi. Croire, ce n'est pas une croyance spirituelle, mais c'est une } \\
\text { croyance qui est matérialisée par ce que vous savez faire. Si vous maîtrisez les éléments, vous } \\
\text { n'avez pas peur ». }\end{array}$ \\
\hline GIPN & $\begin{array}{l}\text { "Nous déprogrammons nos réactions de peur d'abord avec la foi. Si vous ne croyez en rien, } \\
\text { vous n'y allez pas... Les hommes de terrain croient dans le devoir qu'ils doivent remplir vis-à- } \\
\text { vis de la société »; "La motivation, cette fameuse foi, le policier ou le gendarme doit aller la } \\
\text { chercher individuellement, sans aucun encouragement hiérarchique ou social ». }\end{array}$ \\
\hline $\begin{array}{l}\text { Autres } \\
\text { services }\end{array}$ & $\begin{array}{l}\text { "A chaque fois, on a le même profil, phénotype, de personnes qui sont très empruntes de foi. } \\
\text { Elles sont empreintes de foi et empreintes d'une droiture exceptionnelle ». }\end{array}$ \\
\hline RAID & $\begin{array}{l}\text { "Quand on intervient dans la police, on a la loi avec nous, on se sent conforté car on a le } \\
\text { sentiment de faire quelque chose de valable et de justifié. La règle du service est qu'on prend } \\
\text { des risques même pour sauver la vie d'un forcené. C'est l'abnégation. On le sait en entrant »; } \\
\text { "Dans la classe à Neuilly, si ça pète, on est tous morts... On vient tous dans des Services } \\
\text { comme ça pour sauver la veuve et l'orphelin, se dire qu'on fait une bonne action ». } \\
\text { "Le rituel, c'est la préparation du sac, un peu comme en boxe, avec des pratiques non } \\
\text { rationnelles ». }\end{array}$ \\
\hline
\end{tabular}


Nous pouvons avancer que cette « foi » serait en lien avec la motivation intrinsèque, c'est-à-dire la « foi » non pas dans le sens religieux, mais plutôt de conviction dans le bien-fondé de sa mission. Les professionnels interrogés ont des qualités et des compétences rares, et les mettent au service d'une cause: la sécurité nationale, parfois au péril de leur vie. La régulation émotionnelle s'effectue en partie grâce à cette «foi », qui représente une force en chaque professionnel interrogé. D'ailleurs, le terme d'émotion vient du latin «movere»: mettre en mouvement. Sans doute que la « foi » dont il est question est sous-tendue par des émotions décisives, comme la fierté, le sentiment de justice, la joie, l'excitation... Ce rapport à la «foi» suggère que quelque chose de plus fort transcende l'émotion de peur ou de tristesse, dans des contextes extrêmes.

Certains professionnels apprennent à réguler et à gérer leurs émotions grâce à la " mithridatisation », c'est-à-dire l'entraînement à vivre des situations extrêmes. Cet aspect absent dans la revue de littérature, a cependant son importance: si les entraînements recréent les conditions extrêmes réelles, ils jouent un rôle fondamental dans la régulation émotionnelle des policiers d'élite.

Tableau 4: La «mithridatisation », comme moyen de gestion émotionnelle des policiers

\begin{tabular}{|c|c|}
\hline BRI & $\begin{array}{l}\text { "Les entraînements servent à être plus efficace, plus efficient, et donc obligatoirement, } \\
\text { d'abaisser la peur. On maîtrise. S'entraîner, c'est arriver à développer des éléments d'instinct. } \\
\text { On sera plus sûrs de nous, plus confiants, plus solides. Cela va engendrer moins de peurs, de } \\
\text { doutes et d'interrogations, qui sont des émotions négatives. Trop d'émotions négatives peuvent } \\
\text { tuer l'action, car à un moment votre métabolisme physiologique va rentrer en action d'une } \\
\text { manière tellement forte, qu'il va prendre le dessus. Vous serez dans le cas d'un stress aigu. } \\
\text { Vous serez tétanisé, ou vous ferez n'importe quoi. On acquiert les bons comportements par } \\
\text { de l'entraînement. Lorsque vous vous retrouvez dans des situations difficiles en entraînement, } \\
\text { dans du stress, dans le dépassement de soi, ce n'est pas juste pour vous pousser, mais pour } \\
\text { arriver à repousser les frontières, qui feront que le jour où, en intervention, vous serez à une } \\
\text { porte de ces frontières, vous aurez encore de la marge derrière ". }\end{array}$ \\
\hline & $\begin{array}{l}\text { "Gérer la peur, c'est se mithridatiser. Mithridate était un roi de l'Antiquité, qui, pour ne pas } \\
\text { être empoisonné, prenait tous les jours des petites doses de poison pour habituer son corps. } \\
\text { J'ai fait cela toute ma vie. Dès que je craignais quelque chose, je faisais tout pour le connaître, } \\
\text { le gérer... La meilleure façon de gérer sa peur, c'est de se rendre compte que cela ne sert à } \\
\text { rien d'avoir peur sauf de vous handicaper... "; " Je me raisonne et force mon esprit à tenir } \\
\text { compte de paramètres } \\
\text { réels et à taire mon affectif... On rationnalise la peur, le danger... On peut toujours s'habituer } \\
\text { à toutes situations ». } \\
\text { "La sélection, l'entraînement, limitent les risques d'erreur en intervention, car on a toujours } \\
\text { peur de ce qu'on ne connaît pas et l'entraînement permet de se familiariser avec des situations } \\
\text { incongrues ". }\end{array}$ \\
\hline & $\begin{array}{l}\text { "Toutes les phases de préparations, d'exercices et d'entraînements sont poussées. La qualité } \\
\text { des Services d'élite réside pour beaucoup dans le fait qu'ils mènent des entraînements très } \\
\text { poussés ». "Le niveau des entraînements est souvent plus élevé que les conditions réelles ». }\end{array}$ \\
\hline IID & $\begin{array}{l}\text { visibles entre nous. C'est le but de l'entraînement. On sait quand on va } \\
\text { agir ainsi, que celui derrière va agir ainsi etc». }\end{array}$ \\
\hline
\end{tabular}

Les résultats montrent que les professionnels expriment leur peur de ne pas bien faire leur travail: "Ce dont on a le plus peur est de ne pas bien faire notre travail » (RAID). Cette peur provient de l'imprévisibilité de la situation en intervention. Toutes les situations d'interventions étant par nature imprévisibles, les entraînements permettent de maîtriser le plus possible les différents éléments. Enfin, nous découvrons que pour les policiers interrogés, il existe une certaine 
«dépendance à l'adrénaline » ou "addiction », qui est plutôt une forme de dépendance aux situations extrêmes, l'adrénaline étant une des substances sécrétées lors de situations à fortes charges émotionnelles. Cette dépendance peut même se transformer en souffrance, lorsque le professionnel se retire des opérations et n'est plus en service: "Pour moi, les stages de boxe que je donne actuellement sont des 'consolations' au manque! »(RAID). Cet aspect n'est pas mentionné dans la revue de littérature mobilisée.

\subsection{Proposition de modélisation}

Concernant la gestion émotionnelle individuelle, les compétences émotionnelles $\mathrm{du}$ professionnel lui permettent d'effectuer le difficile travail émotionnel. Ce dernier consiste la plupart du temps en un travail émotionnel en profondeur, permis par une forme de régulation émotionnelle: la réévaluation cognitive, consciente. Deux autres formes de gestion émotionnelle individuelle permettent au policier d'élite de réguler ses émotions en intervention: la «foi» et la " mithridatisation ». Grâce à ces deux paramètres, le policier va pouvoir maîtriser le plus possible les éléments de l'intervention, et donc mieux gérer sa peur. $\mathrm{Au}$ niveau collectif, le soutien social contribue à la gestion émotionnelle. C'est le cas aussi de l'humour. Les articulations précédemment décrites entre régulation émotionnelle et travail émotionnel, entre travail émotionnel et compétences émotionnelles, puis entre travail émotionnel, régulation émotionnelle et soutien social, apportent des éléments de réflexion pour optimiser les pratiques de GRH. La modélisation proposée est présentée dans la figure 1.

Figure 1: Modélisation de la gestion des émotions au travail

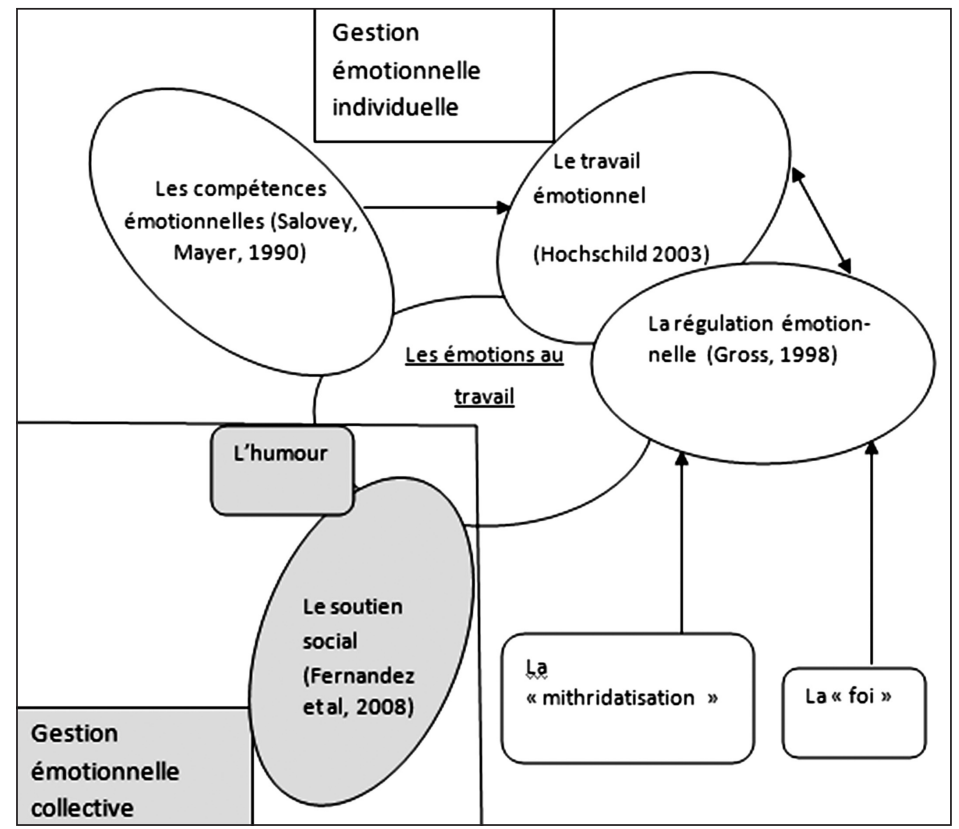




\section{Conclusion}

Cette recherche présente plusieurs limites. Au niveau méthodologique, la difficile généralisation du cas induit une validité externe très relative. Elle ouvre essentiellement de nouvelles perspectives. Des aspects permettant une gestion émotionnelle ont émergé lors des entretiens et mériteraient d'être approfondis. C'est le cas du rôle de l'humour, dont il conviendrait d'analyser la nature, le rôle et le degré. Il permet, semble-t-il, une forme de régulation émotionnelle collective. L'humour ne signifie pas un manque de sérieux dans le travail, mais constitue un véritable sas de décompression. L'évocation de la «foi » par les policiers soulève également des interrogations. La «foi » présenterait quelque intérêt à être cernée, comprise, afin d'en capter les aspects et les conséquences sur la gestion émotionnelle dans ces métiers. Peut-être se rapproche-t-elle du concept de motivation intrinsèque, cette impression de pouvoir faire la différence, grâce à la nature même du travail effectué. Quelle est la nature de cette foi, son rôle, son degré? Nous pouvons aussi nous poser la question de la nécessité d'élaborer des questionnaires de $\mathrm{QE}$, ou d'imaginer des mises en situations d'évaluation des compétences émotionnelles. Le sujet de la reconversion professionnelle mériterait aussi d'être approfondi par les professionnels de la fonction $\mathrm{RH}$, dans ces unités d'élite: qu'en est-il du malaise ou de la souffrance relative au sevrage de cette "addiction » aux situations extrêmes? D'un point de vue plus général, après repérage de bonnes pratiques prenant en considération la gestion des émotions au travail dans les trois niveaux d'analyse, quelles transférabilités des outils et pratiques de GRH paraissent envisageables dans des métiers où la charge émotionnelle fait moins partie intégrante du métier? Par exemple, les téléopérateurs ou les chauffeurs de bus peuvent être confrontés à de la violence, de l'agressivité, et subir de fortes charges émotionnelles au travail. Ces « incidents émotionnels » peuvent survenir alors même que les professionnels en question (chauffeurs de bus, téléopérateurs, enseignants) n'ont pas été préparés et entraînés à vivre et surmonter de telles épreuves, à la différence des policiers d'élite.

L'article aborde la gestion des émotions chez les policiers d'élite sous l'angle de quatre concepts: le travail émotionnel (Hochschild, 2003), la régulation émotionnelle (Gross, 1998) et les compétences émotionnelles (Salovey et Mayer, 1990), concernant les aspects individuels de cette gestion; et le soutien social (Cintas et Sprimont, 2011; La Rocco et Jones, 1978), concernant les aspects collectifs. Pour les policiers rencontrés, travail en profondeur et réévaluation cognitive s'associent, sans lien clairement établi avec les risques sur la santé. Les compétences émotionnelles du professionnel lui permettent d'effectuer le travail émotionnel requis. Le rôle du soutien social comme forme de gestion collective des émotions, apparait essentiel. Au-delà des aspects théoriques initiaux, ont émergé des entretiens le rôle de la «foi », de l'humour, de l'addiction aux situations extrêmes, et de la " mithridatisation ». D'un point de vue opérationnel, la nécessaire prise en compte par la GRH des compétences émotionnelles et du travail émotionnel du personnel, à la fois dans le recrutement, les formations, la gestion des carrières et des compétences, et grâce à une mise en œuvre de conditions favorables au soutien social est soulignée. Il en va de la santé du professionnel et de sa performance en intervention. 


\section{Références :}

Adelmann P.K. (1995), Emotional labor as a potential source of job stress, in Sauter S.L., Murphy L.R. (dir.), Organizational risk factors for job stress, Washington, DC, US, American Psychological Association, p. 371-381.

Allard-Poesi F. (2003), Coder les données, in Giordano Y. (dir.), Conduire un projet de recherche, une perspective qualitative, Caen, EMS, p. 245-290.

Blanchet A., Gotman A. (1992), L'enquête et ses méthodes: l'entretien, Paris, Nathan.

Cintas C., Sprimont P.A. (2011), Soutien social et violence au travail: quels effets sur le burn-out? Laboratoire NIMEC-IAE de Rouen.

Ekman P. (2010), Je sais que vous mentez, Paris, Editions Michel Lafon

Fernandez F., Leze S., Marche H. (2008), Le langage social des émotions: études sur les rapports au corps et à la santé, Paris, Economica-Anthropos.

Giroux N. (2003), L'étude de cas, in Giordano Y. (Coord.), Conduire un projet de recherche, une perspective qualitative, Colombelles, EMS, p. 41-84.

Gross J., Richards J.M., John O. (2006), Emotion regulation in everyday life, in Snyder D., Hughes J., Simpson J. (Dir.), Emotion regulation in couples and families: pathways to dysfunction and health, Washington DC, APA, p.13-35.

Gross J., John O. (2003), Individual differences in two emotion regulation processes: Implications for affect, relationships, and well-being, Journal of personality and social psychology, n ${ }^{\circ} 85$, p. 348362.

Gross J. (2002), Emotion regulation : affective, cognitive, and social consequences, Psychophysiology, n'39, p.281-291.

Gross J. (2001), Emotion regulation in adulthood: timing is everything, Current directions in psychological science, $\mathrm{n}^{\circ} 10$, p. 214-219.

Gross J. (1998), Antecedent and response-focused emotion regulation: divergent consequences for experience, expression, and physiology, Journal of personality and social psychology, $\mathrm{n}^{\circ} \mathrm{74}, \mathrm{p}$. 224237.

Hamel J. (1997), Etude de cas et sciences sociales, Paris, L'Harmattan.

Hamel J., Dufour S., Fortin D. (1993), Case study methods, Newbury Park, CA, Sage Publications.

Hochschild A.R. (2003), The Managed Heart: Commercialization of Human Feeling, University of California Press.

Kotsou I. (2008), Intelligence émotionnelle et management: comprendre et utiliser la force des émotions, Bruxelles, De Boeck.

La Rocco J.M., Jones A.P. (1978), Co-workers and leader support as moderators of stress-strain relationships in work situations, Journal of Applied Psychology, n63, p. 629-634.

Lazarus R. (1991), Emotion and adaptation, Oxford, UK, Oxford University Press.

Loriol M. (2003), La construction sociale de la fatigue au travail: l'exemple du burn-out des infirmières hospitalières, Travail et Emploi, n 94, p. 65-74.

Molinier P. (1998), Psychodynamique du travail et identité sexuelle, thèse de doctorat en psychologie, CNAM.

Rispal M. (2002), La méthode des cas, Paris, De Boek Université.

Ruiller C. (2010), Le soutien social à l'hôpital et son influence sur les comportements, Paris, Editions Presses Universitaires Européennes.

Salovey P., Brackett M.A., Mayer J.D. (2004), Emotional intelligence: Key readings on the Mayer and Salovey model, New York, Dude Press, Port Chester.

Salovey P., Mayer J.D., Caruso D.R. (1999), Emotional intelligence meets traditional standards for an intelligence, Intelligence, $\mathrm{n}^{\circ} 27$, p. 267-298.

Salovey P., Mayer J.D. (1997), What is emotional intelligence? in Salovey P. et Sluyter D.J. (Eds.), Emotional Development and Emotional Intelligence, New York, Basic Book, p.3-31.

Salovey P., Mayer J.D. (1990), Emotional Intelligence, Imagination, Cognition, and Personality, ${ }^{\circ} 9$, p.185-211.

Scherer K.R. (2000), Psychological models of emotion, in Borod J.C. (Eds.), The Neuropsychology of Emotion, New Jork, Oxford University Press, p. 137-162.

Van Hoorebeke D. (2004), La gestion des émotions au travail: droit fondamental du salarié? n683, CEROG, IAE Aix en Provence.

Van Hoorebeke D. (2003), Le management des émotions au travail: une reconsidération des pratiques organisationnelles, Revue de Gestion des Ressources Humaines, n4, p.2-14.

Yin R.K. (2002), Case Study Research, Design and Methods, Newbury Park, Sage Publications. 
Annexe 1: Guide d'entretien des professionnels du RAID

- Présentez-vous, votre parcours, vos tâches principales sur le terrain et en tant que négociateur du RAID.

- Qu'est-ce que le RAID et son rôle, sa place dans les services de l'Etat?

Au poste de travail

- En quoi consiste le travail d'un négociateur du RAID? Quelles sont les compétences émotionnelles principales requises pour un négociateur du RAID?

Pratiques de Gestion des Ressources Humaines

- RECRUTEMENTS : dans les recrutements, dans quelle mesure sont prises en compte ces compétences émotionnelles? Quelle différence de recrutement entre un négociateur et un homme de terrain non-négociateur au RAID?

- FORMATIONS : quelles sont les formations inculquées aux hommes du RAID?

- MANAGEMENT: quel type de management menez-vous en tant que négociateur du RAID? Comment et par qui êtes-vous vous-même managé? Les hommes du RAID communiquent-ils à leur hiérarchie leurs situations à risques, les situations d'émotions déplaisantes importantes, les agressions, les traumatismes émotionnels?

- CONDITIONS DE TRAVAIL et ORGANISATION DU TRAVAIL: Les émotions déplaisantes des hommes du RAID sont-elles parfois liées à leurs conditions de travail?

- GESTION DES RISQUES PSYCHO-SOCIAUX: Des mesures ont-elles été mises en place par les RH afin de gérer les situations stressantes, les émotions déplaisantes des hommes du RAID, les cas de burn-out, de stress post-traumatique?

Les émotions déplaisantes / le stress

- Quelles sont les situations stressantes ou génératrices d'émotions déplaisantes / plaisantes principales pour un homme du RAID? Quelles sont les émotions principales ressenties lors d'une intervention : avant pendant et post-intervention et pourquoi? Comment ces émotions s'expriment-elles physiologiquement? Comment sont-elles gérées? Quels sont les principaux facteurs de stress dans ce métier?

- Face à la mort et au danger, quelle est la place de la peur dans ce métier?

- Quel est le rôle de l'imprévu?

- Selon vous, les hommes du RAID ressentent-ils des difficultés à gérer ces émotions déplaisantes?

- Les hommes du RAID sont-ils victimes de désordres psychosomatiques?

- Comment gérer le juste équilibre entre vie professionnelle et personnelle?

Gestion des émotions et du stress

- Y a-t-il des cas importants de stress, de RPS ou de burn-out?

- Quel est le rôle des médecins du travail et des psychologues du travail?

- Post-intervention: comment évacuez-vous vos émotions et votre stress professionnel?

- Quel est le rôle du dialogue pour évacuer vos émotions? Avec qui évacuez-vous vos émotions par les rapports interpersonnels? De quel type de soutien émotionnel et social bénéficiezvous?

- Existe-il des groupes de paroles formels ou informels? Quelle est leur utilité? Rôle du collectif.

- Selon vous, quels sont les facteurs qui permettent de mieux gérer ses émotions dans ce travail: âge, expérience, personnalité, compétences émotionnelles, etc?

Le travail émotionnel fourni par les hommes du RAID

- Selon vous, le travail émotionnel fourni par les hommes du RAID est-il dépendant de la personne et / ou des conditions de travail et d'organisation du travail?

- Finalement, quel est, selon vous, le travail émotionnel principal fourni par un homme du RAID?

- En quoi ce travail émotionnel peut-il conditionner la qualité des interventions sur le terrain?

- Pensez-vous que ce travail émotionnel soit indispensable afin de mener votre tâche à bien dans ce métier? 
Annexe 2: Tableau des résultats au niveau individuel, collectif et de la fonction $\mathrm{RH}$

\begin{tabular}{|c|c|}
\hline \multicolumn{2}{|r|}{ Formes de gestion émotionnelle individuelle } \\
\hline $\begin{array}{c}\text { Travail } \\
\text { émotionnel } \\
\text { et régulation } \\
\text { émotionnelle }\end{array}$ & $\begin{array}{l}\text { "Nous sommes dans un jeu en profondeur... Je me conditionne au calme. Quand il y a des } \\
\text { coups de feu... je dois renvoyer une image de calme et de maîtrise. Du coup, je suis convaincu } \\
\text { intérieurement que je suis dans cette condition de calme. Si c'ết été un jeu en surface, c'eût été } \\
\text { décelable » (négociateur du RAID). } \\
\text { "Quand on a affaire à des populations qu'on sait être auteurs de délits... Il faut jouer un jeu » } \\
\text { (autres services de sécurité de l'Etat). } \\
\text { A l'intérieur de l'équipe: « Quand il y a de la casse... on ne peut pas le montrer. C'est affreux, } \\
\text { car personne ne doit voir que vous êtes affecté " (RAID). } \\
\text { Impacts sur la santé: "Par exemple, un médecin légiste confronté deux ou trois fois de suite à } \\
\text { des enfants, va finir par présenter un certain nombre de lésions, c'est normal (autres services } \\
\text { de sécurité de l'Etat); "Oui, on le fait, mais cela ne me coûte pas. C'est inclus dans le contrat» } \\
\text { (RAID). }\end{array}$ \\
\hline $\begin{array}{l}\text { Communication } \\
\text { non-verbale }\end{array}$ & $\begin{array}{l}\text { "Il faut d'abord trouver une parade, avoir l'air naturel, car eux vont me voir. Il va falloir que } \\
\text { vous sortiez de votre personne, tout en restant dedans. Il faut mettre une fausse façade devant le } \\
\text { personnage " (BRI). } \\
\text { "Immanquablement, nous finissions par voir passer une, deux ou trois personnes, dont } \\
\text { l'attitude, le comportement général, nous faisaient nous retourner l'un vers l'autre et hocher de } \\
\text { la tête: nous avions découvert nos cibles 》(GIPN). } \\
\text { Empathie non seulement exprimée, mais ressentie "On doit comprendre la logique inhérente à } \\
\text { la personne, qui n'est pas la nôtre. C'est une empathie complète. Il faut rentrer dans son système } \\
\text { de pensées » (autres services de sécurité de l'Etat). }\end{array}$ \\
\hline $\begin{array}{l}\text { Régulation des } \\
\text { émotions }\end{array}$ & 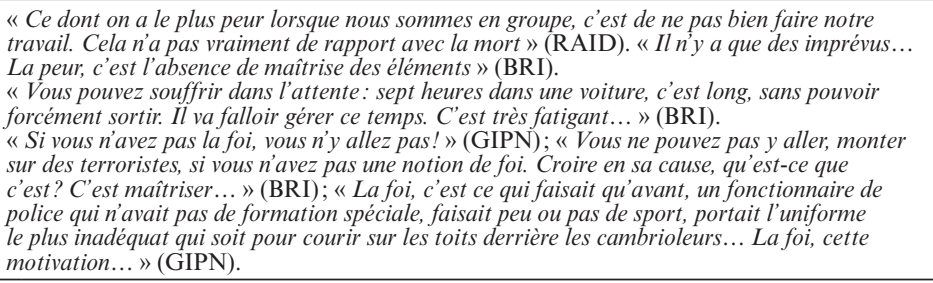 \\
\hline $\begin{array}{l}\text { Formes de } \\
\text { retraits }\end{array}$ & $\begin{array}{l}\text { La tristesse générée par la mort d'un collègue: émotion la plus intense. } \\
\text { Les policiers d'unités d'élite restent rarement toute leur carrière dans des unités telles que le } \\
\text { RAID, la BRI, le GIPN, ou le GIGN. } \\
\text { Avoir la « juste distance » (Loriol, 2003, p.6): « Pour être professionnel et efficace, il faut } \\
\text { comprendre le forcené pour le désamorcer. Il faut le gérer. On ne se perd pas dans l'affectif, on } \\
\text { n'a pas le droit » (GIPN). } \\
\text { Traumatisme émotionnel: « A Ris-Orangis, celui qui a été grièvement blessé a vu ses deux } \\
\text { collègues mourir devant lui, dont un qui est mort sur lui. Il donnait l'impression d'être fiable } \\
\text { mais en fait il était friable... Il a fini par partir car il ne tenait plus » (RAID). }\end{array}$ \\
\hline \multicolumn{2}{|r|}{ Type de soutien social perçu } \\
\hline Collègues & $\begin{array}{l}\text { "Briefs et debriefs d'intervention », plus ou moins formels: « Les autres nous connaissent } \\
\text { exactement sur ce que nous sommes... 》 (RAID); « Au GIGN, le système de fonctionnement } \\
\text { est basé avant tout sur de l'empirisme, donc les retours d'expériences à chaud et à froid } \\
\text { y sont très importants. Le but est d'avoir du retour d'expériences, d'apprendre. Le sas de } \\
\text { décompression et de cohésion se fait généralement au restaurant, après une opération. Ce sas } \\
\text { est quelque chose qui ne peut être évité » (autres services de sécurité de l'Etat). } \\
\text { Pendant l'intervention: « Je suis bien parce que le fait que tout le monde me regarde et } \\
\text { craigne pour moi fait que je me sentais bien. On me dit que j'ai dû avoir chaud, mais en fait } \\
\text { non!» (RAID, durant une mise en joue par le forcené). }\end{array}$ \\
\hline Humour & $\begin{array}{l}\text { "Quand on blague, on blague! Il y a toujours des choses drôles et des anecdotes... Cela } \\
\text { permet de passer des choses au second degré... C'est une manière de décompresser, et de } \\
\text { prendre des situations moins au sérieux, se jouer des situations négatives » (RAID). }\end{array}$ \\
\hline Hors travail & $\begin{array}{l}\text { "On ne ramène pas cela avec nous... On cloisonne. C'est le maître-mot. On apprend à fermer } \\
\text { la porte quand on sort... Il s'agit là d'une règle d'or » (autres services de sécurité de l'Etat). }\end{array}$ \\
\hline $\begin{array}{l}\text { Reconnaissance } \\
\text { sociale }\end{array}$ & $\begin{array}{l}\text { «Ce qui m’interpelle quand je discute avec ces gens-là... et que je parle de mon métier, c'est } \\
\text { qu'ils sont fascinés par ma vie. Ils me disent que ce que je fais est le métier dont ils rêvaient » } \\
\text { (BRI). }\end{array}$ \\
\hline Psychologues & $\begin{array}{l}\text { "Il n’y a pas vraiment de suivi psychologique chez nous... Les personnes estiment que le psy } \\
\text { n'y connait rien à l'histoire, il n'a jamais été au charbon avec un calibre à la main » (RAID). }\end{array}$ \\
\hline Management & $\begin{array}{l}\text { «Parfois, de la part de notre Direction, on a une volonté de ne pas venir nous féliciter... On } \\
\text { peut en souffrir, au niveau de la reconnaissance au travail »(BRI); « Le gradé, si on nest pas } \\
\text { au boulot, est comme les autres, se fait charrier, etc. En intervention, il redevient le chef de } \\
\text { service en tant que tel... »(RAID). }\end{array}$ \\
\hline
\end{tabular}




\begin{tabular}{|c|c|c|}
\hline \multicolumn{3}{|c|}{ Pratiques de GRH prenant en compte les compétences émotionnelles des policiers d'élite } \\
\hline & Métiers de négociateur & Métiers de policier opérationnel \\
\hline Recrutement & \multicolumn{2}{|c|}{$\begin{array}{l}\text { La Direction des RH des unités d'élite prend en compte ces compétences, } \\
\text { mais rarement de manière formelle: "Il n'y a pas de test de QE. En fait, } \\
\text { empiriquement, on en avait. Les différentes mises en situation étaient des tests de } \\
\text { QE, mais on ne les avait pas nomenclaturés tels quels. Demain, il serait peut-être } \\
\text { très utile d'en créer » (RAID). } \\
\text { Les compétences se mesurent grâce à des exercices physiques, pour détecter } \\
\text { la personnalité et la force mentale des candidats: « Toutes les phases de } \\
\text { préparations, d'exercices et d'entraînements sont poussées » (autres services de } \\
\text { sécurité de l'Etat); " Dans les tests de combat par exemple, on va tout de suite } \\
\text { voir celui qui est capable d'essayer de s'organiser, se protéger, ou se cacher et être } \\
\text { soumis » (RAID). } \\
\text { " Sang-froid, adaptation, discernement, prises de décisions, initiatives, maîtrise } \\
\text { de soi, compétences interpersonnelles, empathie... », tests, simulations, mises en } \\
\text { situation. }\end{array}$} \\
\hline Forn & $\begin{array}{l}\text { «Il faut un Master II } \\
\text { en négociation de crise } \\
\text { et une bonne formation } \\
\text { théorique, axée en partie } \\
\text { sur le relationnel ». Le } \\
\text { négociateur étudie: « De la } \\
\text { sociologie, de la psychologie, } \\
\text { de la criminologie, de la } \\
\text { victimologie, de l'ethnologie, } \\
\text { de l'éthologie... » (RAID). }\end{array}$ & $\begin{array}{l}\text { "Malheureusement, avec les problèmes financiers, } \\
\text { on nous refuse des stages et des formations. C'est } \\
\text { de la GRH... " (BRI). } \\
\text { Robert Paturel, professionnel reconnu sorti des } \\
\text { services: « Moi je n'ai jamais assisté à un cours } \\
\text { de gestion du stress au RAID! Parce que j’étais } \\
\text { le premier à en parler ». Il indique qu'il n'a pu } \\
\text { assister à aucune formation sur la maîtrise du } \\
\text { stress au RAID durant toute sa carrière. } \\
\text { La BRI note aussi un gros manque de formation } \\
\text { à la gestion du stress et des émotions déplaisantes, } \\
\text { à la gestion du temps en planque. }\end{array}$ \\
\hline Entraînements & $\begin{array}{l}\text { se mithridatiser. Mithridate é } \\
\text { empoisonné, prenait tous les } \\
\text { corps. J'ai fait cela toute ma } \\
\text { pour le connaître, le gérer... } \\
\text { à tenir compte de paramètres } \\
\text { danger... On peut toujours s' }\end{array}$ & $\begin{array}{l}\text { « la mithridatisation »: " Gérer la peur, c'est } \\
\text { ait un roi de l'Antiquité, qui, pour ne pas être } \\
\text { urs des petites doses de poison pour habituer son } \\
\text { ie. Dès que je craignais quelque chose, je faisais tout } \\
\text { (GIPN). «... Je me raisonne et force mon esprit } \\
\text { t à taire mon affectif... On rationnalise la peur, le } \\
\text { abituer à toutes situations » (GIPN). }\end{array}$ \\
\hline
\end{tabular}

